JOURNAL OF THEORETICAL

AND APPLIED MECHANICS

57, 4, pp. 833-842, Warsaw 2019

DOI: $10.15632 /$ jtam-pl/112017

\title{
COMPUTATIONAL DOMAIN DISCRETIZATION FOR CFD ANALYSIS OF FLOW IN A GRANULAR PACKED BED
}

\author{
MARCIN SOSNOWSKI \\ Jan Dlugosz University in Czestochowa; e-mail: m.sosnowski@ujd.edu.pl \\ Renata Gnatowska \\ Czestochowa University of Technology; e-mail: gnatowska@imc.pcz.czest.pl \\ JACEK SOBCZYK, WALDEMAR WODZIAK \\ Strata Mechanics Research Institute of the Polish Academy of Sciences \\ e-mail: sobczyk@img.pan.krakow.pl
}

\begin{abstract}
The paper reports results of an analysis concerning the infuence of a computational domain discretization method on the numerical stability of a model as well as the calculation error. The topology of a packed bed of a granular material consisting of granules contacting tangentially in one point makes the modeling of heat and mass transfer due to the fluid flow in such a domain a chalenging task. Therefore, the contribution of this paper constitutes a summary of discretization methods with discussion and guidelines allowing one to effectively select the most favourable method dedicated to discretization of the domain. The validation using Particle Image Velocimetry and evaluation of the impact of inflow velocity on the experimental and numerical research results are also presented in the paper.
\end{abstract}

Keywords: granular materials, computational domain, discretization, CFD

\section{Introduction}

The granular packed beds of materials are the subject of many research due to their widespread use in several engineering applications and various industries (Asendrych and Niegodajew, 2017; Niegodajew et al., 2018; Suekane et al., 2003; Szymanek and Tyliszczak, 2018). The numerical methods such as Computational Fluid Dynamics (CFD) have proved their usefulness as an effective research tool for industry and can be successfully used in flow analysis concerning granular material beds (Sosnowski et al., 2018; Vargas and McCarthy, 2001).

In the numerical modeling methods, the inlet conditions and discretization of the computational domain is a critical stage, because it affects not only the accuracy of the results but also the calculation time and numerical stability of the model (Gnatowska, 2019).

Low numerical diffusivity is the main advantage of the hexahedral mesh, but generation of this type of mesh for complex geometries is difficult or even impossible (Fig. 1). Generation of the tetrahedral mesh is relatively simple, but the results obtained with the tetrahedral mesh are imposed to errors resulting from high diffusivity of mesh elements. The polyhedral mesh is a beneficial compromise as it combines acceptable numerical diffusivity with ease of generation. The advantage of this mesh is that each individual cell is adjacent to others. While, for the tetrahedral mesh, the neighboring cells are in contact with only four elements. The polyhedral mesh contributes to better approximation of gradients, which in turn leads to more reliable results of CFD calculations. In addition, polyhedral cells are less sensitive to stretching compared to tetrahedral cells, which improves the numerical stability of the calculation. 

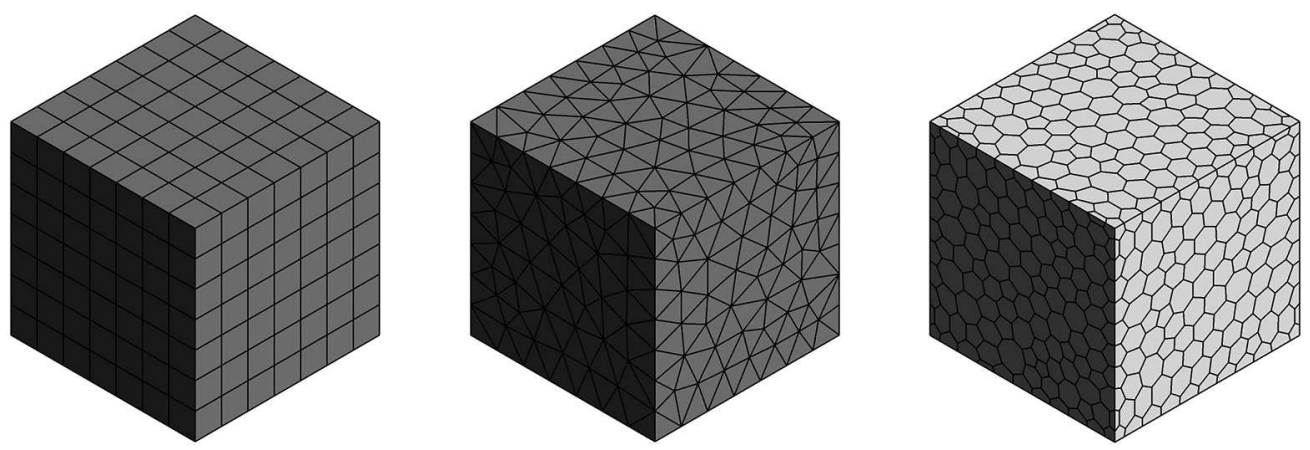

Fig. 1. Types of mesh: hexahedral - left, tetrahedral - center, polyhedral - right (Sosnowski et al., 2018)

The quality of the mesh is important while modelling a flow field in granular packed beds due to the specific topology of the domain in which individual granules contact tangentially each other at a single point.

\section{Methods}

Three spheres of diameters equal to $30 \mathrm{~mm}$ each were the experimental research object. They represented granules located along a line and contacted tangentially. The granules were mounted on a wooden stand (Fig. 2a). The experimental research was carried out with the use of the Particle Image Velocimetry (PIV) method in the aerodynamic wind tunnel as depicted in Fig. 2b. The free stream velocity of the tunnel varied from 0.1 to $62 \mathrm{~m} / \mathrm{s}$, and the longitudinal free stream turbulence intensity was less than $0.5 \%$ (Bujalski et al., 2014). The systems dedicated to control temperature and relative humidity was installed in the wind tunnel. The PIV system included of 5.5Mpx sCMOS camera and Nd:YAG laser emitting a pair of $0.2 \mathrm{~J}$ light pulses. The flow was seeded with $\sim 1 \mu \mathrm{m}$ droplets of Di-Ethyl-Hexyl-Sebacate (DEHS). The plane through the centers of the three granules was illuminated with a laser light sheet. Double frame images were recorded at $15 \mathrm{~Hz}$ frequency rate, and an adaptive correlation algorithm was used in the analysis.

CFD analysis of the computational domain representing the granular material is a challenging task due to the pre-processing issues that concern discretization of the domain where the individual granules contact each other in a single point and also due to very narrow spaces to be meshed in the direct vicinity of the above mentioned contact points (Fig. 3a). This yields numerical instability of the model resulting directly from the excessively low quality grid elements. Researchers usually solve this issue by reducing the radius of the modelled granules with preserving their original locations (Fig. 3b) - such an approach was called the granule radius reduction (GRR) method (Sosnowski et al., 2018). The main drawback of this method is the disturbance of flow and heat transfer in the analyzed domain. Another approach to discretization of a granular material domain consists in the extension of the contact point between the two individual granules to a cylindrical volume as shown in Fig. 3c. It was investigated in (Bu et al., 2014; Sosnowski et al., 2018) and named the contact point extension (CPE) method (Sosnowski et al., 2018). This approach allows one to discretize the computational domain with a high-quality mesh and simultaneously maintain geometrical contact between individual granules.

The applied type of mesh elements is another important issue influencing the CFD results and directly associated with computational domain discretization (Sosnowski et al., 2018). In consequence, four cases were investigated within the numerical research: 2 methods (granule radius reduction, contact point extension) and mesh types (tetrahedral, polyhedral). The applied boundary conditions are shown in Fig. 4. It represents one quarter of the experimental research object depicted in Fig. 2 placed in a virtual wind tunnel. The dimensions of the wind tunnel 

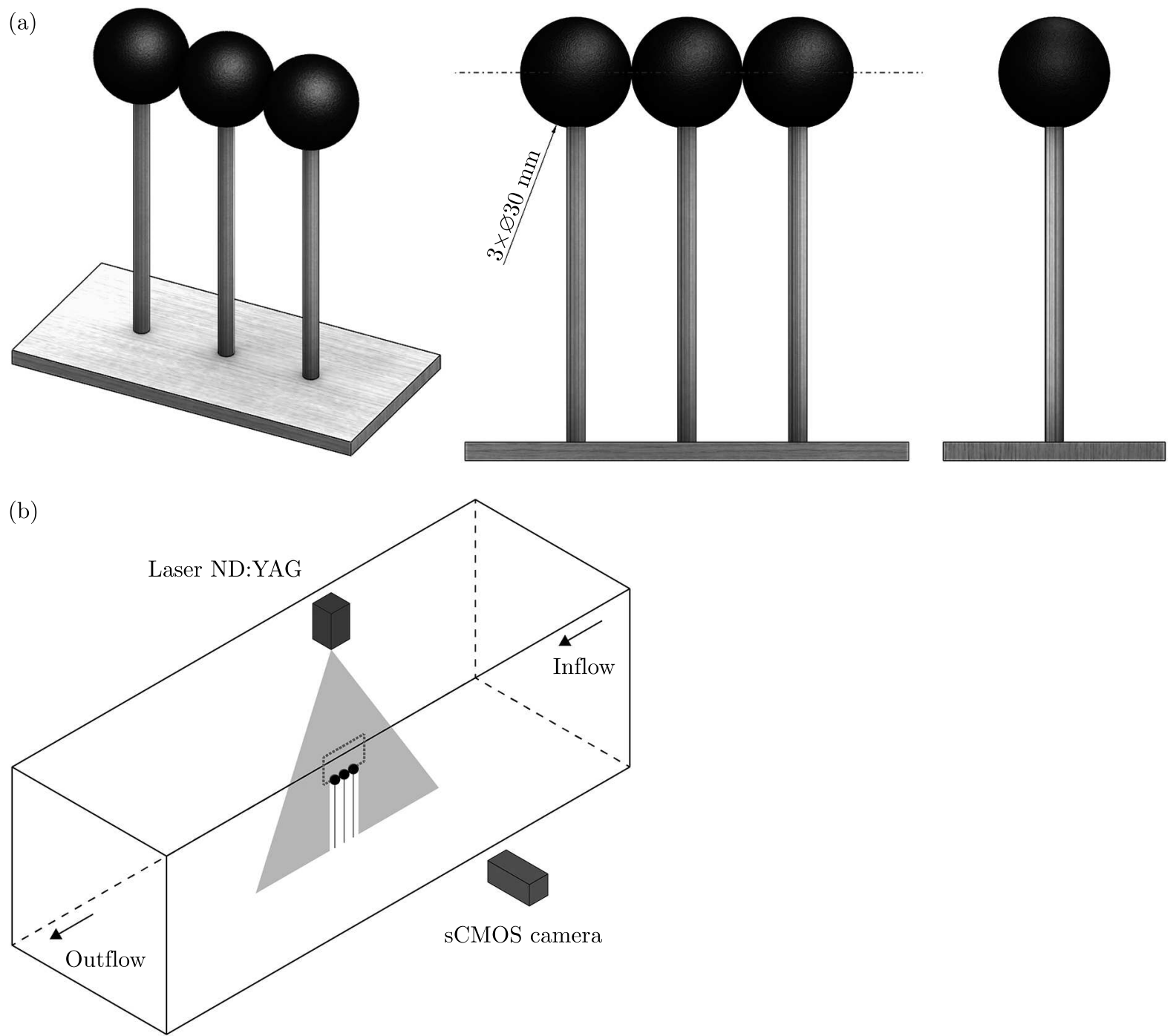

Fig. 2. Scheme of the research object (a) and experimental stand (b) (Sosnowski et al., 2018)

(a)

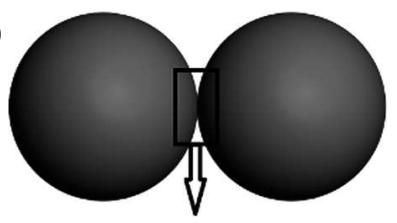

(b)

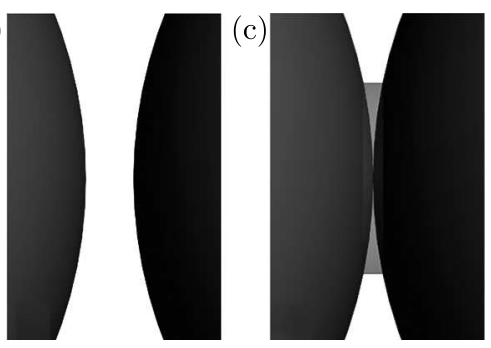

Fig. 3. The methods of granules contact representation: (a) contact point method, (b) granule radius reduction (GRR) method, (c) contact point extension (CPE) method (Sosnowski et al., 2018) 
domain are based on the best practice guidelines. The cylinder radius was equal to $r / 5$ defined as optimal according to (Bu et al., 2014; Sosnowski et al., 2018).

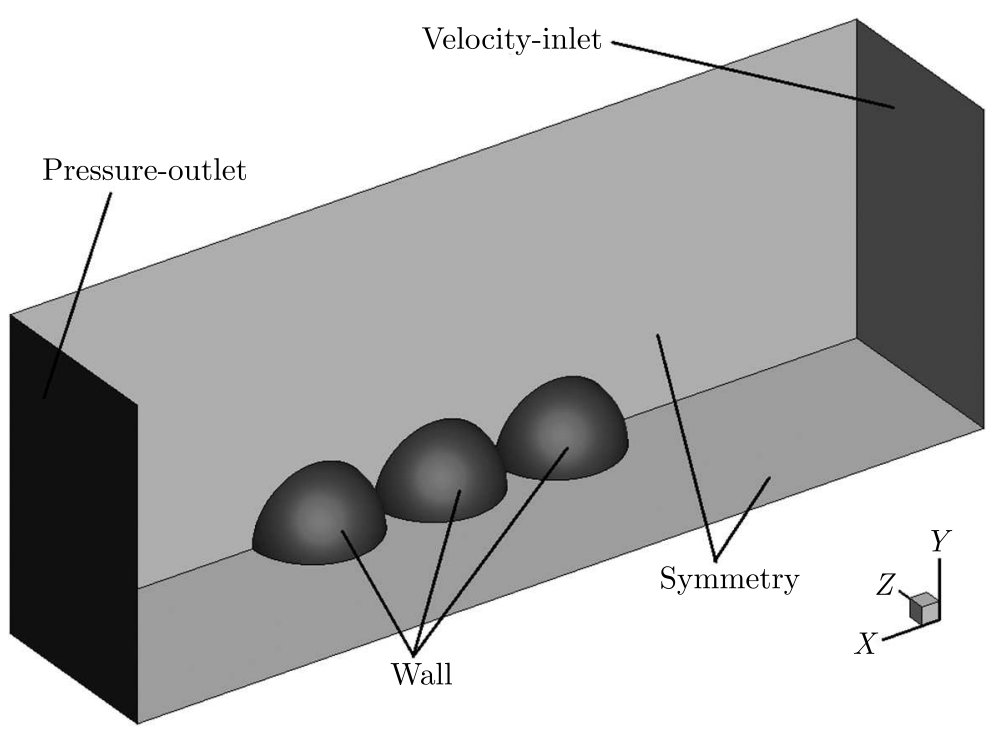

Fig. 4. Applied boundary conditions (Sosnowski et al., 2018)

The inlet boundary condition of the computational domain was defined as the velocity-inlet (Fig. 4). The inlet velocities were defined based on the experimental research conditions. The section planes depicted in Fig. 4 were set as symmetric. The beginning of the $x$ and $y$ axis were located in the middle in the contact between the 1 st and the 2 nd granule. The standard $k-\varepsilon$ viscous model was applied in simulations.

The complete and detailed description of the experimental research and numerical method was presented in the previous work (Sosnowski et al., 2018).

\section{Results and discussion}

The experimental PIV research was carried out for three inlet velocities $(0.62 \mathrm{~m} / \mathrm{s}, 1.81 \mathrm{~m} / \mathrm{s}$ and $4.20 \mathrm{~m} / \mathrm{s}$ ) and the numerical research were performed for twelve configurations: three inlet velocities corresponding with the experiment, two discretization methods and two mesh types.

Mesh quality factors concerning the mesh used in CFD analysis are presented in Table 1. The minimum orthogonal quality ranges from 0 to 1 ( 0 corresponds to low quality; 1 corresponds to the ideal maximum aspect ratio). The mesh quality factors calculated for the investigated configurations indicate a very good quality of POLY and good quality of TETRA.

The values of all residuals below the level of $1 \mathrm{e}-3$ were considered as the indication of numerical convergence of the model. In the last three rows (Table 1), the number of iterations necessary to gain model convergence was at least two times higher in the case of TETRA in comparison to POLY for all inflow velocities. In the case of lower values of inflow velocities, the advantage of polyhedral over tetrahedral in terms of convergence was even more significant. It leads to a conclusion that POLY is preferable also in terms of time. The number of iterations to convergence was not affected by the method of representation contact point of the granules.

Figures 5-7 depict the velocity profiles for all investigated inflow velocities, methods and mesh types recorded along a line located $2 \mathrm{~mm}$ above the granules, which corresponds to $17 \mathrm{~mm}$ above the line crossing through the contact points of the granules. As it can be seen, the CFD results for analyzed inflow velocities are qualitatively and quantitatively similar regardless of the contact point representation method. Some minor discrepancies are revealed in terms of the 
Table 1. Mesh quality factors and model convergence

\begin{tabular}{l|c|c|c|c|c|}
\cline { 2 - 5 } \multicolumn{2}{c|}{} & \multicolumn{2}{c|}{ Contact point extension } & \multicolumn{2}{c|}{ Granule radius reduction } \\
\cline { 2 - 6 } \multicolumn{2}{c|}{} & poly mesh & tetra mesh & poly mesh & tetra mesh \\
\hline \hline \multicolumn{2}{|l|}{ Minimum orthogonal quality } & 0.52 & 0.17 & 0.68 & 0.16 \\
\hline \multicolumn{2}{l|}{ Maximum aspect ratio } & 5.53 & 19.70 & 5.16 & 21.86 \\
\hline \multirow{2}{*}{$\begin{array}{l}\text { Iterations } \\
\text { to } \\
\text { convergence }\end{array}$} & $V=0.62 \mathrm{~m} / \mathrm{s}$ & 166 & 416 & 167 & 421 \\
\cline { 2 - 6 } & $V=1.81 \mathrm{~m} / \mathrm{s}$ & 153 & 364 & 154 & 364 \\
\cline { 2 - 6 } & $V=4.20 \mathrm{~m} / \mathrm{s}$ & 160 & 300 & 162 & 304 \\
\hline
\end{tabular}

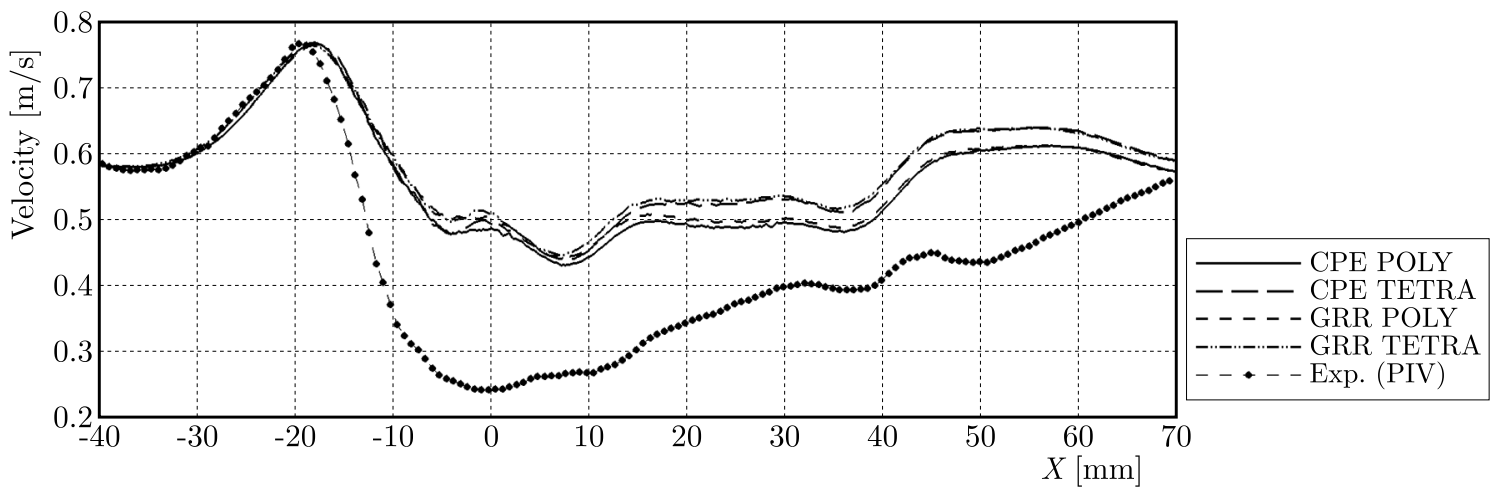

Fig. 5. Velocity profiles along a line located $2 \mathrm{~mm}$ above granules $(y=17 \mathrm{~mm})$ for inflow velocity equal to $0.62 \mathrm{~m} / \mathrm{s}$

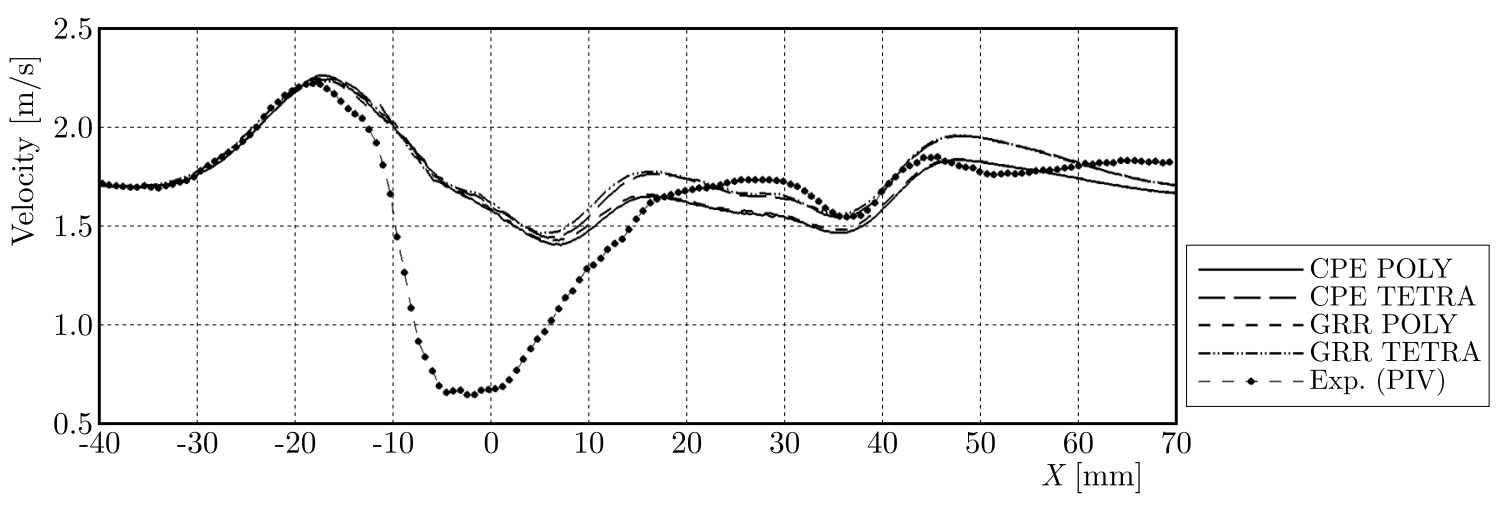

Fig. 6. Velocity profiles along a line located $2 \mathrm{~mm}$ above granules $(y=17 \mathrm{~mm})$ for inflow velocity equal to $1.81 \mathrm{~m} / \mathrm{s}$

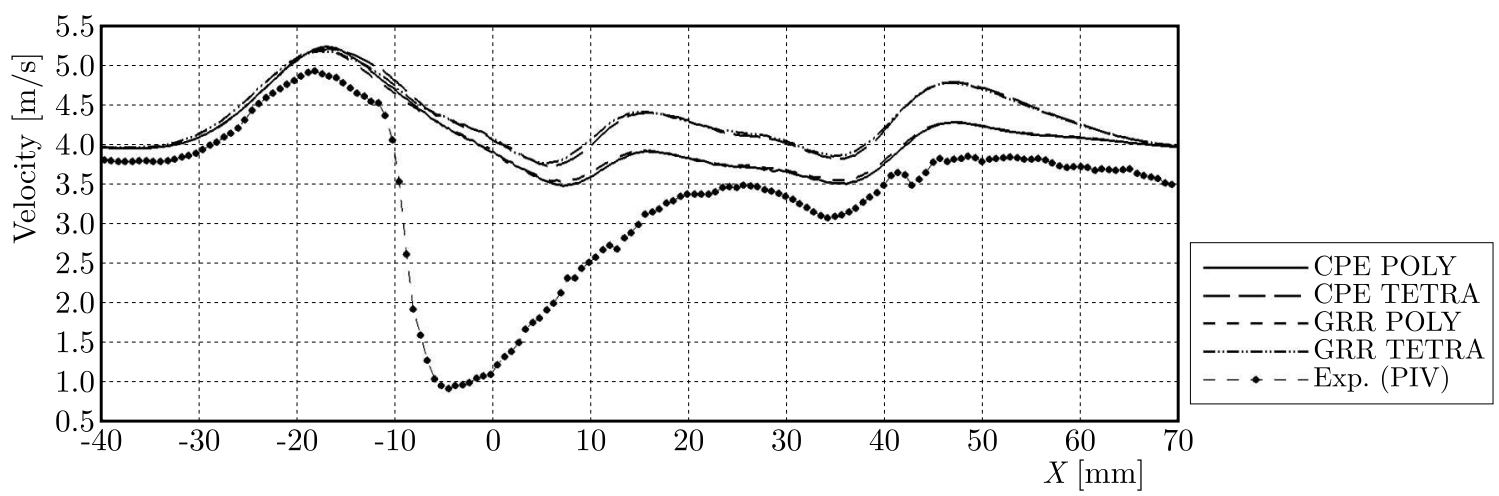

Fig. 7. Velocity profiles along a line located $2 \mathrm{~mm}$ above granules $(y=17 \mathrm{~mm})$ for inflow velocity equal to $4.20 \mathrm{~m} / \mathrm{s}$ 
applied mesh type - tetrahedral mesh overestimates the velocity magnitude although the results are still qualitatively similar to the results obtained with the polyhedral mesh. The comparison of CFD and PIV results reveals more significant qualitative differences, especially in the vicinity of the first granule. It can be caused by an insufficient boundary layer representation in the numerical model or low PIV resolution equal to $0.712 \mathrm{~mm}$. Therefore, further analysies need to be carried out in order to prove the said thesis. The CFD results of the three analyzed inflow velocities are qualitatively comparable. The same applies to the PIV results, except for the case of inflow velocity equal to $0.62 \mathrm{~m} / \mathrm{s}$, which differs from the other results.

Time-averaged velocity fields achieved during the experimental research with the use of PIV and CFD numerical calculations for the analyzed cases are presented in Figs. 8-10. The figures present both a wide view of the three granules and a close-up view of the gap between the first and the second granule. The flow fields for both views are presented in different scales in order to precisely evaluate the velocity near the contact point of granules.

The applied method of contact point representation does not significantly affect the flow field in the direct vicinity of the granules, but the applied mesh type impacts the results more significantly. It results from the lower numerical diffusion of the polyhedral mesh and it is revealed in reduced velocity fluctuations. This issue is clearly visible along the vertical axis of the computational domain after the third granule. Moreover, it intensifies along with the increase of inflow velocity. Therefore, the flow field obtained with the application of the polyhedral mesh are more consistent with the PIV experimental results, which proves the impact of the applied mesh type on the CFD results.

The close-up view indicates differences between PIV and CFD results. CFD overestimates the local velocity in the direct vicinity of the contact point in the case of inflow velocity equal to $0.62 \mathrm{~m} / \mathrm{s}$ and underestimates it for inflow velocity equal to $4.20 \mathrm{~m} / \mathrm{s}$. The CFD results for the case of $1.81 \mathrm{~m} / \mathrm{s}$ inflow velocity best correspond with PIV results, although the results for the granule radius reduction method along with the tetrahedral mesh differs from the other cases.

The influence of contact point representation method on the obtained results can only be seen in the contact region occupied by the additional cylindrical volume generated with the contact point extension method. The flow velocity magnitude $3 \mathrm{~mm}(r / 5)$ upwards from the granules contact point does not exceed $0.0002 \mathrm{~m} / \mathrm{s}$ in the case of the inflow velocity equal to $0.62 \mathrm{~m} / \mathrm{s}, 0.02 \mathrm{~m} / \mathrm{s}$ for the inlet velocity $1.81 \mathrm{~m} / \mathrm{s}$ and $0.30 \mathrm{~m} / \mathrm{s}$ for the inlet velocity $4.20 \mathrm{~m} / \mathrm{s}$. It is, respectively, $0.03 \%, 1.1 \%$ and $7.1 \%$ of the inflow velocity, which indicates a non-linear relationship between the local velocity near the contact point and the inflow velocity.

\section{Conclusions}

The carried out research concerning the impact of the inflow velocity, granules contact point representation and the numerical mesh type on the flow field within a packed bed of a granular material proves that each of the said factors have to be carefully considered in order to gain reliable results.

The application of both methods (the contact point extension; the granule radius reduction) does not significantly influence the flow above the granules, but the reduction of granule radius (in the granule radius reduction method) considerably disturbs the calculated porosity of the bed of the granular material $-3.26 \%$ error for the granule radius reduction method and $0.03 \%$ error for the contact point extension method.

The used method of granule contact representation does not affect significantly the mesh quality factors. 

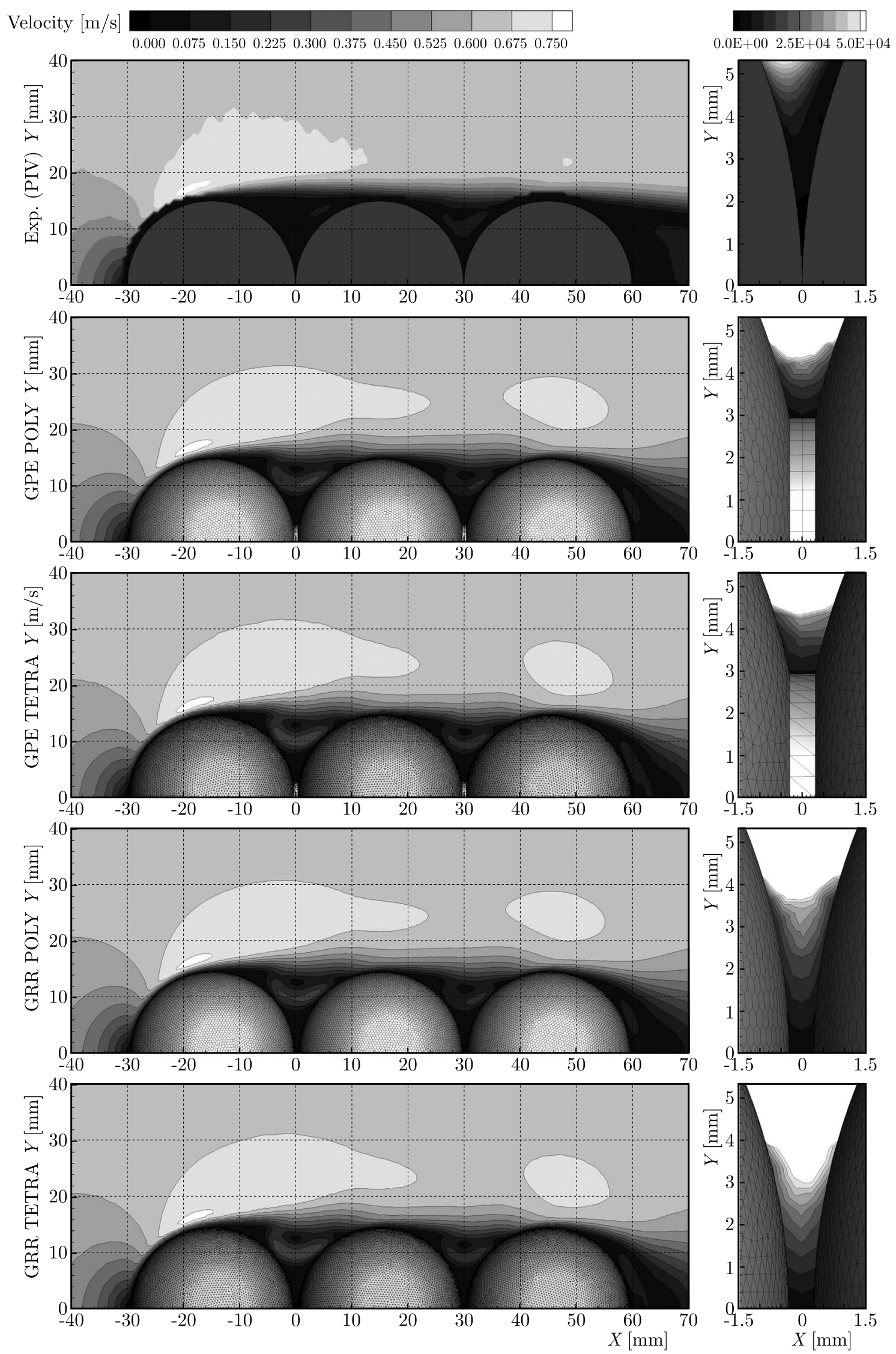

Fig. 8. Time-averaged velocity field for inflow velocity equal $0.62 \mathrm{~m} / \mathrm{s}$ 

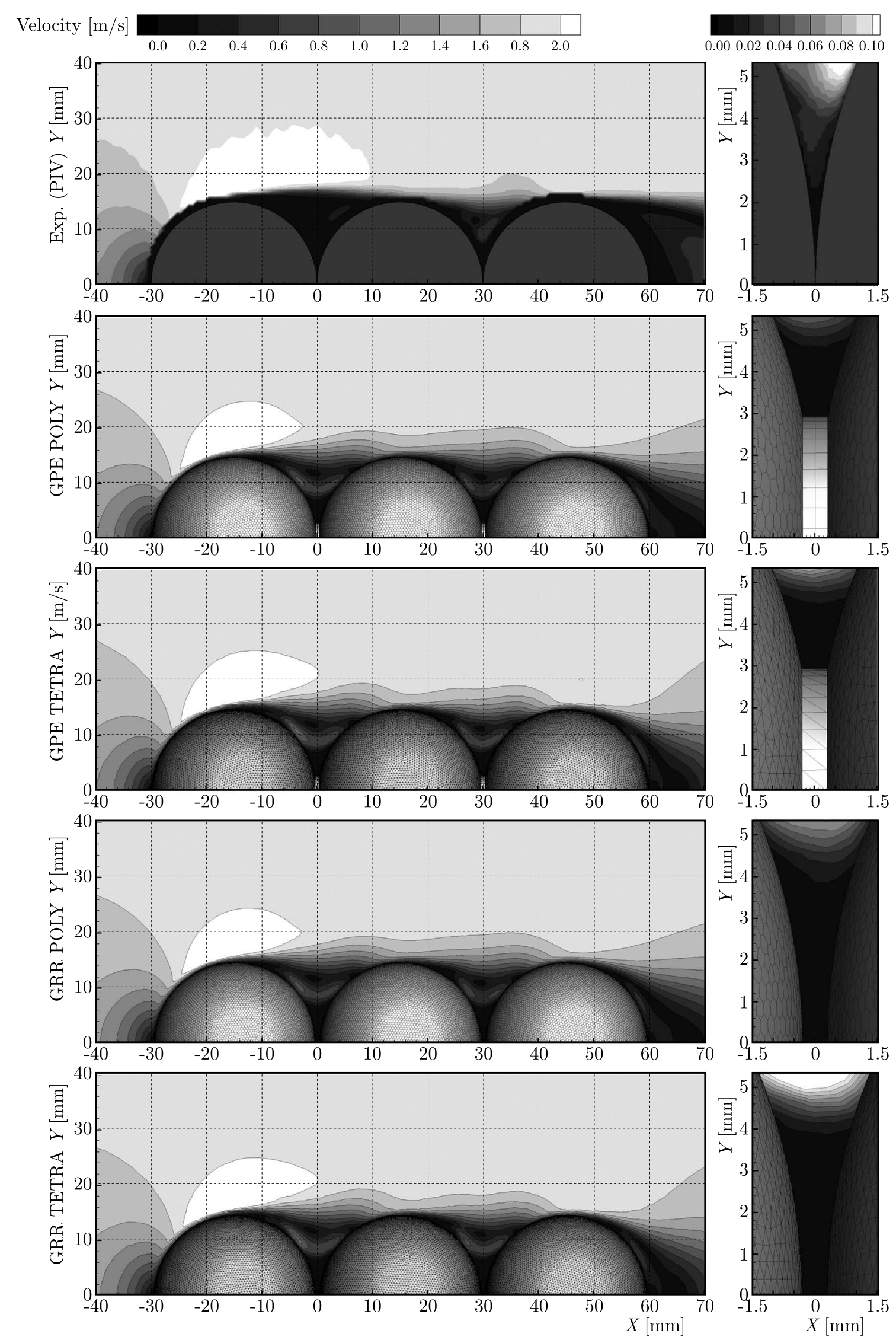

Fig. 9. Time-averaged velocity field for inflow velocity equal $1.81 \mathrm{~m} / \mathrm{s}$ 

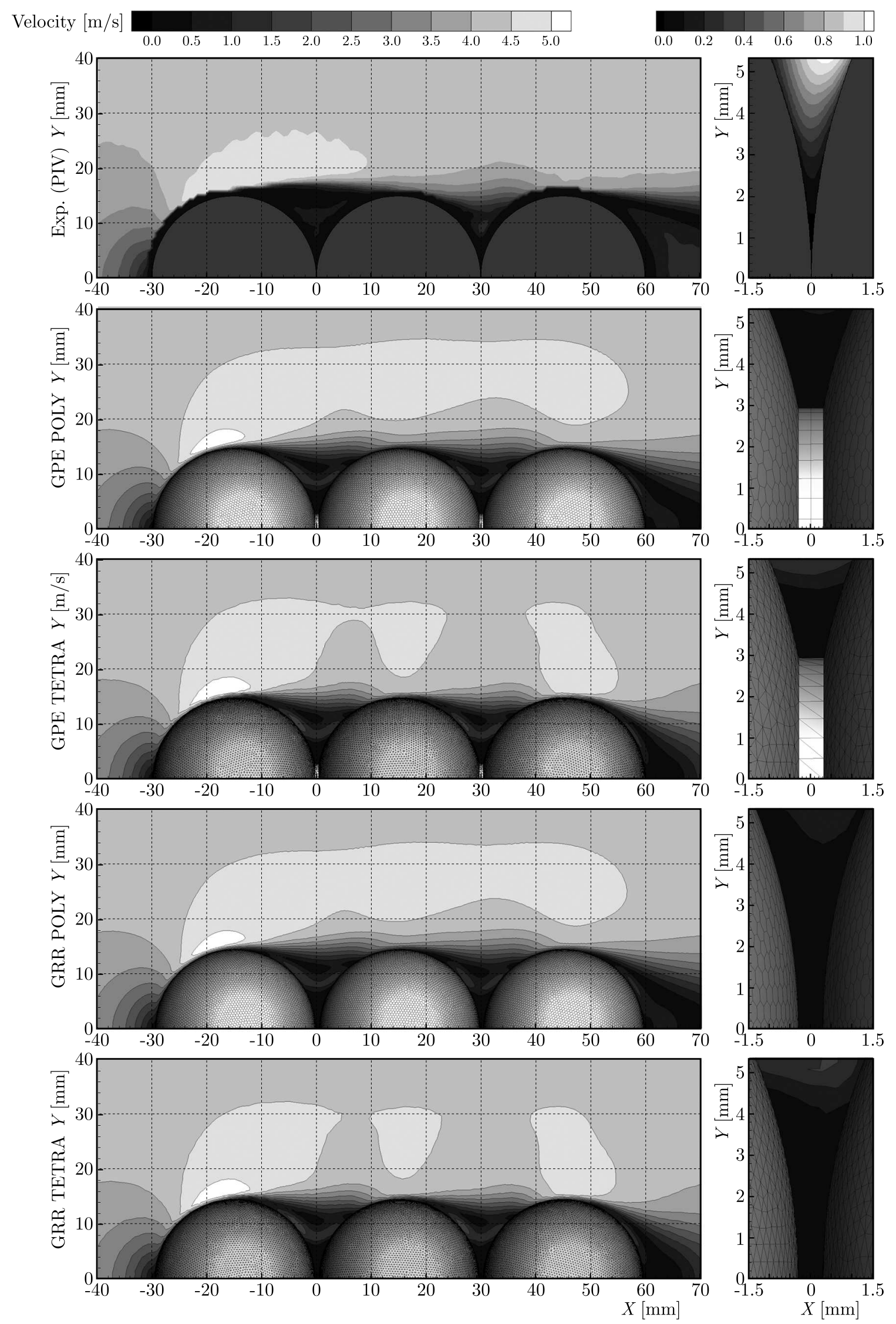

Fig. 10. Time-averaged velocity field for inflow velocity equal $4.20 \mathrm{~m} / \mathrm{s}$ 
The advantages of the polyhedral mesh are revealed in a more accurate boundary layer representation and a better agreement of numerical calculations and experimental results. These advantages result from the improved characteristics of polyhedral elements in terms of calculation diffusivity. Moreover, the convergence is obtained within a lower number of iterations for the polyhedral mesh (advantage for industrial applications).

The inflow velocity influences the consistency of experimental and numerical results mostly in the contact region of the granules. The local velocity is underrated by CFD in comparison to PIV in the case of higher inflow velocity, and overrated in the case of lower inflow velocity. The contact point extension method with POLY as the preferable mesh type is the recommended method for discretization of the computational domain representing a granular packed bed regardless of the inflow velocity magnitude.

Acknowledgements

Supported by Polish National Centre of Science 2017/01/X/ST8/00019 and 2017/01/X/ST8/00076.

\section{References}

1. Asendrych D., Niegodajew P., 2017, Numerical study of the $\mathrm{CO}_{2}$ absorber performance subjected to the varying amine solvent and flue gas loads, Chemical Engineering Communications, 204, 5, 580-590

2. Bu S., Yang J., Zhou M., Li S., Wang Q., Guo Z., 2014, On contact point modifications for forced convective heat transfer analysis in a structured packed bed of spheres, Nuclear Engineering and Design, 270, 21-33

3. Bujalski M., Gawor M., Sobczyk J., 2014, Flow disturbance due to presence of the vane anemometer, Journal of Physics: Conference Series, 530, 012045

4. Celik I.B., Ghia U., Roache P.J., Freitas Ch.J., Coleman H., Raad P.E., 2008, Procedure for estimation and reporting of uncertainty due to discretization in CFD applications, Journal of Fluids Engineering - Transactions of the ASME, 130, 7

5. Gnatowska R., 2019, Wind-induced pressure loads on buildings in tandem arrangement in urban environment, Environmental Fluid Mechanics, 19, 699, DOI: 10.1007/s10652-018-9646-0

6. Niegodajew P., Wilczynski M., Marek M., Drobniak S., Asendrych D., Elsner W., Gnatowska R., Stempka J., 2018, A study of liquid spreading in laboratory scale random packing column with an optical method supplemented with liquid holdup characteristics, Experimental Thermal and Fluid Science, 96, 162-168

7. Sosnowski M., Gnatowska R., Sobczyk J., Wodziak W., 2018, Numerical modelling of flow field within the packed bed of granular material, Journal of Physics, 1101, 012036

8. Sosnowski M., Krzywanski J., Grabowska K., Gnatowska R., 2018, Polyhedral meshing in numerical analysis of conjugate heat transfer, EPJ Web of Conferences, 180, 02096

9. Suekane T., Yokouchi Y., Hirai S., 2003, Inertial flow structures in a simple-packed bed of spheres, AIChE Journal, 49, 1, 10-17

10. Szymanek E., Tyliszczak A., 2018, Experimental and numerical research on heat and air flow through a granular material, Journal of Physics: Conference Series, 1101, 1, 012043

11. Vargas W.L., MCCarthy J.J., 2001, Heat conduction in granular materials, AIChE Journal, $47,5,1052-1059$ 